\title{
The research and design of orthodontic platform framework Weiguo Zhang ${ }^{1, a}$, Sudong Cui ${ }^{2, b}$
}

\author{
${ }^{1}$ Department of Computer Science and Technology, Xi'an University of Science and Technology, \\ Xi'an, 710054, China \\ ${ }^{2}$ Department of Computer Science and Technology, Xi'an University of Science and Technology, \\ Xi'an, 710054, China \\ aemail: zhangwg@xust.edu.cn, bemail: bruce_tender@163.com
}

Keywords: SSM framework; tooth orthodontic; RESTful architecture; MVC

\begin{abstract}
In view of the data sharing in orthodontic industry is not timely, correction case can not be shared, data dispersion problem, combined with MVC design pattern, software hierarchical structure, taking use of container framework Spring, integrated SpringMVC[1][2], Mybatis[3] framework, designed the dental orthodontic information service platform[4], and the url accessing based on SpringMVC partially embody RESTful[5][6] architecture style. The platform can realize data network management and reach the purpose of data sharing and updating in time in orthodontic field.
\end{abstract}

\section{Introduction}

With the development of orthodontic technology in our country in recent years, dental orthodontic information level also need to constantly improve. Orthodontic information of computer aided orthodontic system in traditional $\mathrm{C} / \mathrm{S}$ mode comes from many regions and different departments, which is scattered and can not be updated in time, and taking use of manual processing is not realistic. In this paper, we use $\mathrm{B} / \mathrm{S}$ [7] structure development model and SSM framework to build a dental orthodontic information service platform framework. The J2EE platform development technologies we adopted have super technical ideas and advantages than other development environment. The platform can make full advantage of the convenience, real-time, accuracy of the internet to promote tooth orthodontic to a wider application level. The platform framework presented in this paper can bring some certain reference value to other areas in improving the degree of information and other aspects.

\section{Demand Analysis}

The functional requirements of teeth orthodontic information service platform as shown in Figure 1.

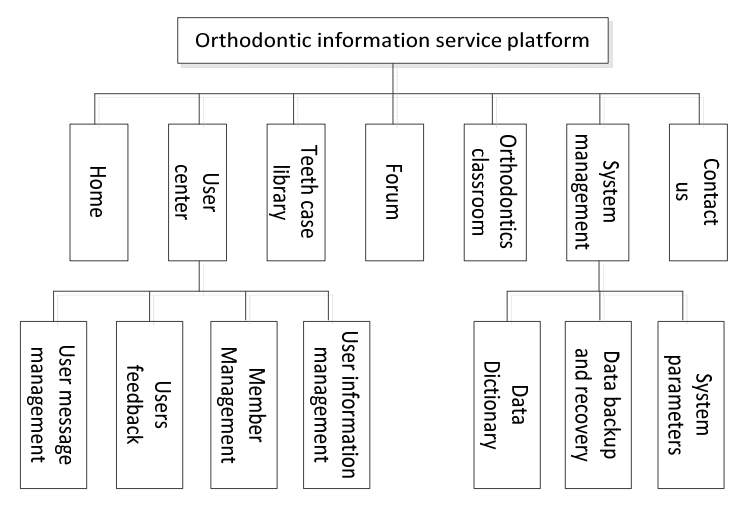

Fig.1. The functional requirements of teeth orthodontic information service platform

(1) Home page: Having a display of the home page, the latest information, dental orthodontics technology, cooperative information displaying, system information, user registration and landing and other related module information. 
(2) User center: Including user registration and landing, dynamic message, dynamic evaluation, friends management, user collection, membership center, system bulletin, post management, user information management and other related functions.

(3) Teeth case library: Referring to the display of case, case upload, information analysis, case retrieval, case download management(general resources, quality resources) and other related functions.

(4) Forum: With the functions of post publication, navigation, topic classification management, post management.

(5) Orthodontics classroom: Providing users with the functions of online video learning and downloading video.

(6) System management: Mainly have database back-up and date restore, system parameter configuration and other related functions.

(7) Contact us: Providing users and manufactures with the channel to contact us.

\section{Design of Orthodontic Platform Framework}

\section{Dental Orthodontic Platform Framework Structure.}

The information framework of tooth orthodontic platform is based on the software hierarchical structure, B/S development model, MVC(Model-View-Control) multi-layer architecture design pattern, SpringMVC[8][9], Mybatis persistence layer framework and open source framework Spring, which is a three layer structure. As is shown in Figure 2.

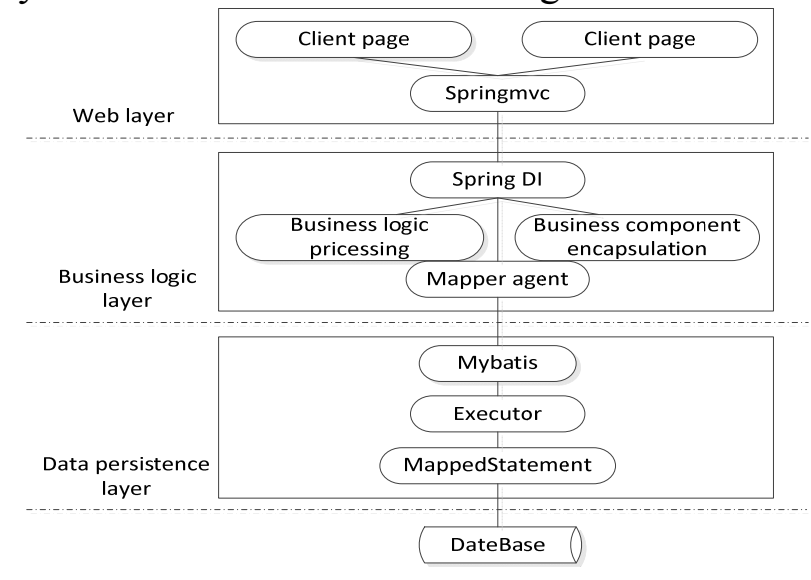

Fig.2. The framework of tooth orthodontic platform

Web layer: SpringMVC[10] as a controller to receive and response user requests has function of controlling page logic. The front controller--DispatcherServlet distributes user requests, requests HandlerMapping find matching handler according to url name or annotation. According to the handler has been found before, HandlerAdapter request Handler processor to perform corresponding handler. SpringMVC can convert Http request into domain object or pojo object with parameter binding mechanism, complete the connection with service layer at the bottom and return the result, which has been rendered, to the client.

Business logic layer: The business logic layer deal with specific business logic, including two parts: interface and implementation. The Spring DI mechanism can reduce the coupling between components, so as to achieve the purpose of decoupling between layers of software and separation of interface and implementation(Spring advocates interface programming). Spring needs to configure dependencies of components in the XML file.

Date persistence layer: The data persistence layer uses mapper agent development model, in which DAO encapsulates all access to data sources. Mybatis focus on SQL statement itself, needing programmers to write SQL statements themselves. Mybatis is an incomplete ORM framework, but it can also realize mapping(input mapping, output mapping).

Mysql was selected as the database, because it has the advantages of open source, small size, fast speed and low cost. 


\section{Key Technology.}

(1) SpringMVC technologies. SpringMVC acts as a control layer in the Web project. It is an excellent web development framework, which is composed of the front controller, processor mapping, processor adapter and view parser. It is a typical textbook type of MVC architecture, receiving the user request and returning the results, achieving system response with other layers. Its functional structure diagram is shown in Figure 3.

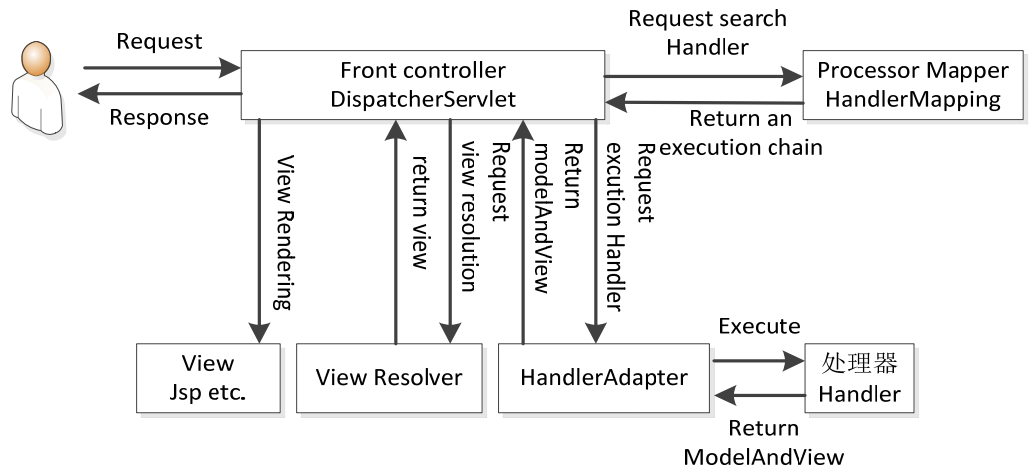

Fig.3. The functional structure diagram of SpringMVC

(2) Spring DI mechanism. Spring is a lightweight Java Web development container framework, which has many advantages, can provide lots of services, such as transaction management, message services, etc. The DI mechanism uses the Java reflection mechanism underling to organizes JavaBeans, in which someone has a dependency on others, configured in the XML file, so that can provide services for the external system. Spring implements the integration between the presentation layer, the controller layer, the business logic layer and the data persistence layer through declaring dependencies between the classes in the configuration file.

(3) Mybatis technology. Mybatis is a persistence layer framework, typical O/R mapping tool, which mainly focus on the SQL statement. Mybatis can automatically map the parameters to be inputted to the preparedStatement into the input, and map the query result set into the Java object(output mapping). Its functional architecture is shown in Figure 4.

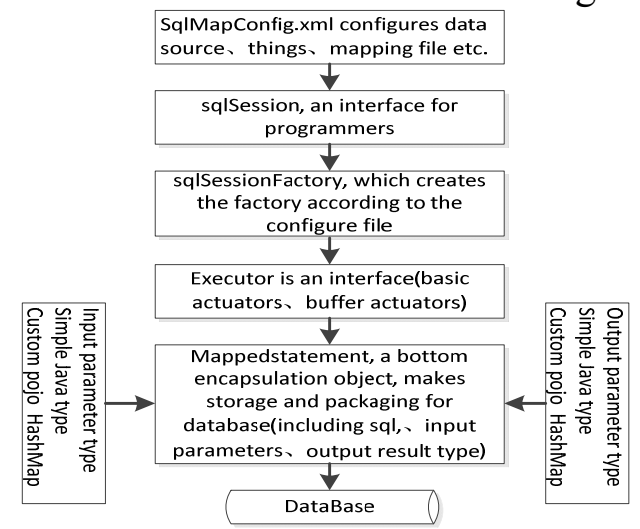

Fig.4. The functional architecture of Mybatis

Framework Development Process.

The dental orthodontic platform framework processing flow is shown in Figure 5.

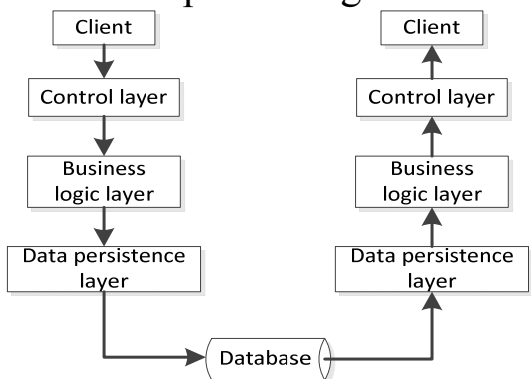

Fig.5. The dental orthodontic platform framework processing flow 
The software development based on software hierarchical structure has the characters of clear division of labor and loose coupling, it can realize the goals of hierarchical development of the presentation layer, control layer, business logic layer and data persistence layer. The specific development process of the framework is as follows:

(1) Using Mybatis's reverse engineering to generate POJO classes and mapper.xml files corresponding to the database table.

(2) The org.mybatis.spring.mapper.MapperScannerConfigurer class in the integrated package mybatis_springmvc automatically scan all the mapper.xml files into the SqlMapConfig file.

(3) Using Dao development pattern to implement the interaction with database in the data persistence layer. Because it is the integration of the SSM framework, the class in this layer does not need to be inherited from the SqlSessionDaoSupport class.

(4) The control layer uses springmvc's annotation tag @controller, @requestMapping to achieve the mapping between Handler and URL, if the match succeeds, the corresponding Hander (there is only one way) will be executed.

\section{Conclusion}

In this study, dental orthodontic platform framework has been designed and laid a good foundation for future detailed design, functional analysis and smooth development. Web development has the characters of covering a wide range, having many technical difficulties, so it needs step by step and specific tasks should be distributed to implement, but a good architecture can give a great convenience to the development and expansion of the system.

\section{References}

[1] XUE F, LIANG F, XU S, et al. Research on SpringMVC framework based Web and its application [J][J]. Journal of Hefei University of Technology (Natural Science), 2012, 3: 013.

[2] Feng Xiao, Feng Lian, Shuxuan Xu, etc. Web research and application based on SpringMVC framework [J]. Journal of HeFei University of Technology: Natural Science Edition, 2012, 35(3): 337-340.

[3] Reddy K S P. Java Persistence with MyBatis 3 [M]. Packt Publishing Ltd, 2013.

[4] Xiaoyan Zhang, Panliang Cai. The information framework research of large coal enterprise of transportation and sale [J]. Industrial automation, 2014(4)

[5] Bing Pan. Research on RESTful Web application oriented resources [J]. Micro computer application, 2010, 31(7): 38-43.

[6] BoWen Zhao. Analysis RESTful Web Service architecture [J]. CD-ROM technology, 2008(10): 4,7 .

[7] Yunyun Li. Simple talk about B/S and C/S architecture [J]. Friends of Science, 2011(1): 6-7.

[8] Wen $\mathrm{Xu}$, Jianhua Gao. Research on Web application framework based on SpringMVC and Mybatis [J]. Micro computer application, 2012(7)

[9] LIU J, DAI J. Research of lightweight Web application based on SpringMVC and iBATIS frameworks $[\mathrm{J}][\mathrm{J}]$. Journal of Computer Applications, 2006, 4: 026.

[10] Mak G. SpringMVC Framework[M] Spring Recipes. Apress, 2008: 321-393 